\title{
Embedding, Clustering and Coloring for Dynamic Maps*
}

\author{
Yifan $\mathrm{Hu}^{\dagger}$ \\ AT\&T Labs - Research \\ Florham Park, NJ
}

\author{
Stephen G. Kobourov \\ Department of Computer Science \\ University of Arizona, Tucson, AZ
}

\author{
Sankar Veeramoni ${ }^{\S}$ \\ Department of Computer Science \\ University of Arizona, Tucson, AZ
}

\begin{abstract}
We describe a practical approach for visualizing multiple relationships defined on the same dataset using a geographic map metaphor, where clusters of nodes form countries and neighboring countries correspond to nearby clusters. Our aim is to provide a visualization that allows us to compare two or more such maps (showing an evolving dynamic process, or obtained using different relationships). In the case where we are considering multiple relationships, e.g., different similarity metrics, we also provide an interactive tool to visually explore the effect of combining two or more such relationships. Our method ensures good readability and mental map preservation, based on dynamic node placement with node stability, dynamic clustering with cluster stability, and dynamic coloring with color stability.
\end{abstract}

Index Terms: G.2 [Discrete Mathematics]: Graph Theory[H.3]: Information Storage and Retrieval—Clustering

\section{Introduction}

Maps offer a familiar way to present geographic data (continents, countries, states). Contact graphs, where regions represent nodes and edges are represented by the corresponding regions sharing borders, are an example of map representations. By definition, contact graphs are limited to planar graphs, but the notion of a map representation can be generalized to non-planar graphs as follows: clusters of well-connected nodes form countries, and countries share borders when neighboring clusters are interconnected. In the context of graph drawing, such maps allow us to show not only connectivity information in the underlying data (via nodes and edges between them), but also clustering (via countries). Specifically, by grouping nodes into different colored regions, we can easily see individual clusters and relations between the clusters. Such explicit grouping makes it easy to identify central and peripheral clusters, as well as central and peripheral nodes within clusters. Finally, cutnodes and edges, often on the border between two clusters, make it clear which nodes and edges connect disparate parts of the data.

Dynamic map visualization deals with the problem of effectively presenting relationships as they change over time. Traditionally, dynamic relational data is visualized by animations of node-and-link graphs, in which nodes and edges fade in and out as needed. One of the main problems in dynamic visualization is that of obtaining individually readable layouts for each moment in time, while at the same time preserving the viewer's mental map. A related problem is that of visualizing multiple relationships on the same dataset. Just as with dynamic data, the main problem is guaranteeing readability while preserving the viewer's mental map. Representations based on the geographic map metaphor could provide intuitive and appealing visualization for dynamic data and for multiple relationships on the same dataset.

\footnotetext{
${ }^{*}$ Research funded in part by NSF grants CCF-0545743, CCF-1115971.

$\dagger$ e-mail: yifanhu@ research.att.com

¥e-mail: kobourov@cs.arizona.edu

§e-mail: sankar@cs.arizona.edu
}

Our motivation comes from population genetics, where one of the major problems is better understanding of the genomic and evolutionary factors shaping human variation and testing models of human origins. Relatively inexpensive human genome sequencing technology allows for the study of patterns of variation across the entire genome in multiple human populations. When genetic information passes down from parents to children some special parts of the DNA remain virtually unaltered as they pass from parent to child. One such piece carrying paternal information is encoded in the non-recombining portion of the Y chromosome (NRY). Another piece carrying maternal information is encoded in the mitochondrial DNA (mtDNA). When comparing DNA information from a group of people, the mtDNA similarity score, the NRY-DNA similarity score, and others can be computed from different parts of the DNA. Such scores can give an indication about the strength of the genetic similarity between the individuals. Interactively combining these multiple scores in an intuitive and visually appealing fashion, facilitates knowledge discovery and hypothesis testing.

With this in mind, our first goal is to provide a way to compare two or more maps of the same objects, obtained using different similarity metrics. Our second goal is to provide an interactive tool to visually explore the effect of combining such similarity metrics. The first problem is related to simultaneous graph drawing, and the second problem is related to dynamic graph drawing. However, in our setting we have to deal not only with the traditional problems of finding suitable placement of nodes and edges, but also with the new problems of dynamic clustering and dynamic color assignment; see Fig. 1. Thus the main contributions of the paper are the following: first, we describe a heuristic to promote dynamic cluster stability; second, we propose an optimal color assignment algorithm to maximize color stability between maps; third, we analyze the use of affine transformation to improve layout stability. Finally, we briefly describe an interactive tool for DNA visualization that utilizes these results.

\section{Related Work}

Our work builds on the GMap algorithm [22], which visualizes relational data and clustering information using a map metaphor. The map metaphor has also been used for information visualization in the cartographic community [15]. Cortese et al. [9] use a topographical map metaphor to visualize prefixes propagation in the Internet, where contour lines describing the propagation are calculated using a force directed algorithm. Also related is work on visualizing subsets of a set of items using geometric regions to indicate the grouping. Byelas and Telea [7] use deformed convex hulls to highlight areas of interest in UML diagrams. Collins et al. [8] use "bubblesets," based on isocontours, to depict multiple relations among a set of objects. Simonetto et al. [30] automatically generate Euler diagrams which provide one of the standard ways, along with Venn diagrams, for visualizing subset relationships. The emphasis of such prior work is on highlighting sets of objects using the map metaphor, rather than on visualizing dynamic maps.

Initial work on dynamic maps was presented in the context of visualizing trends on Internet radio station last. fm [25], where mental map is preserved by fixing node positions. A contextpreserving dynamic text data visualization by Cui et al. [11], keeps the layout stable using a spring embedding algorithm, aided by a mesh constructed over existing nodes. Neither of these two ap- 


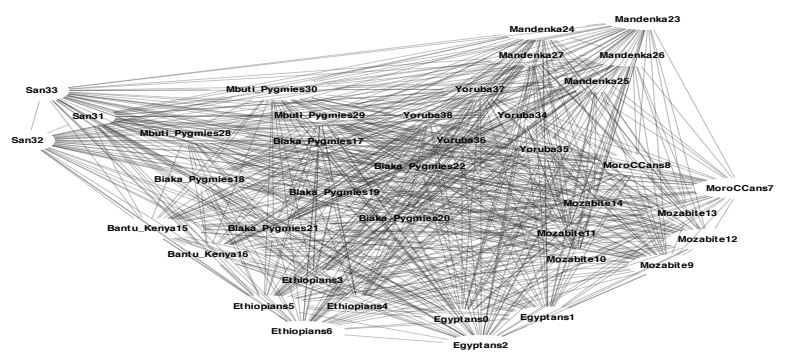

(a)

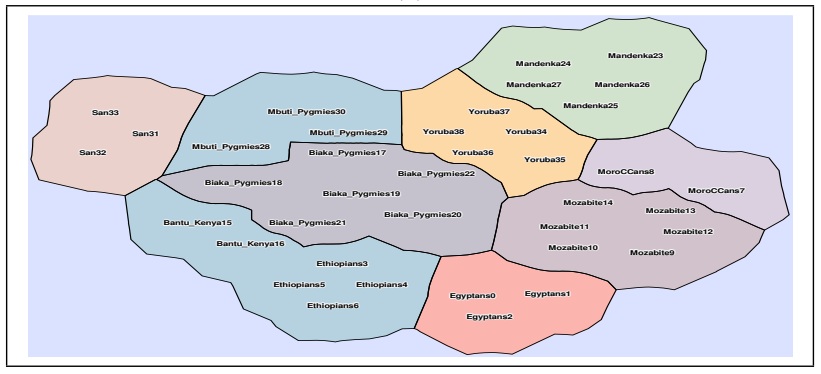

(b)

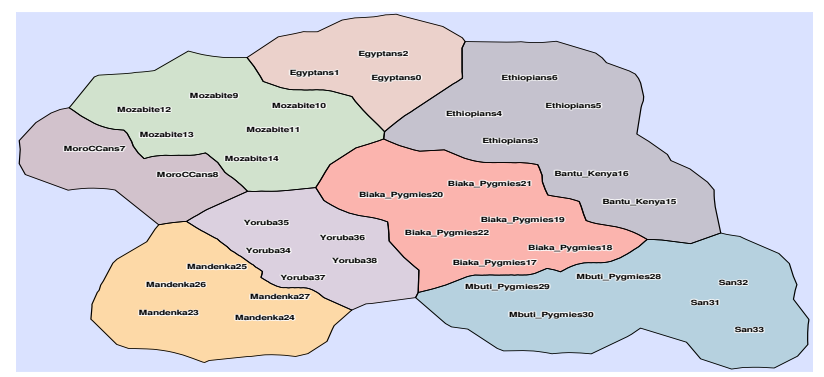

(c)

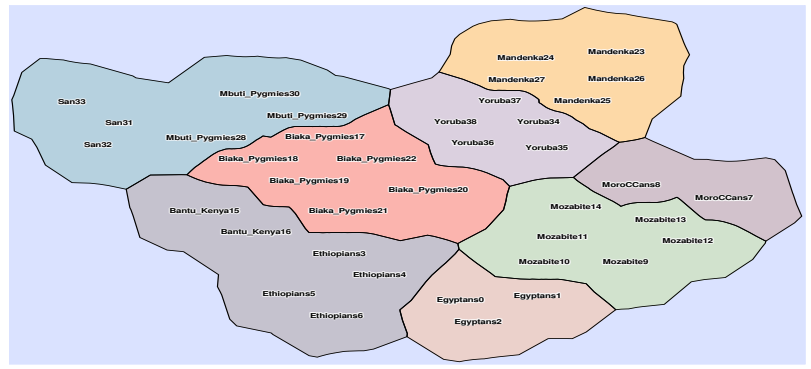

(d)

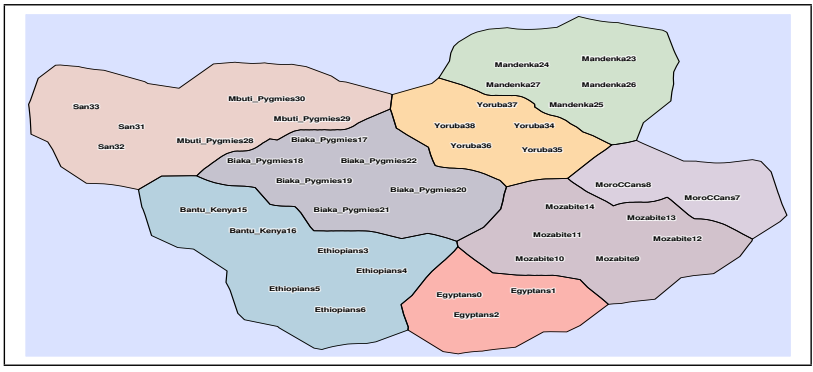

(e)

Figure 1: Visualizing multiple maps requires both layout stability and color stability. (a): mtDNA similarity visualized as a graph. (b): mtDNA similarity visualized as a map. (c): NRY DNA similarity map which is difficult to compare with (b) as the node layout is computed independently. (d): NRY DNA similarity map, computed to optimize node layout stability with regard to (b), which makes is possible to compare nodes, while clusters are still hard to compare. (e): NRY DNA similarity map with optimal node layout and color assignments, which makes it easy to compare with (b); e.g., it is clear that two clusters in the top left of (b) are now merged.

proaches takes cluster and color stability under consideration, either because it is not relevant (in text data), or because clusters are fixed (in last.fm visualization).

Holten and van Wijk [21] study the visual comparison of hierarchically organized data, and propose a method that highlights the differences. Similarly, the TimeRadarTrees of Burch and Diehl [6] are proposed to visualize the evolution of compound digraphs. Although these two approaches deal only with directed graphs and cannot be applied directly to our problem, they are related to the problem of highlighting similarities and differences in dynamic data, which is the aim of this paper.

Also related to our work is a large body of work on dynamic graph layout, where the goal is to maintain a nice layout of a graph that is modified via operations such as inserting/deleting edges and inserting/deleting nodes. Brandes and Wagner adapt the forcedirected model to dynamic graphs using a Bayesian framework [4], while Herman et al. [20] rely on good static layouts. Diehl and Görg [12] consider graphs in a sequence to create smoother transitions. Brandes and Corman [2] present a system for visualizing network evolution in which each modification is shown in a separate layer of a $3 \mathrm{D}$ representation, with nodes common to two layers represented as columns connecting the layers. Thus, mental map preservation is achieved by pre-computing good locations for the nodes and fixing the position throughout the layers. While the issue of mental map preservation adopted in such approaches remains an important one for us, we note that in our application we want to avoid a distortion of the layout for the purpose of mental stability. This is because such distortions introduce biases that were not in the data itself, which is not important when visualizing the evolution of citation graphs or Internet graphs, but are undesirable when analyzing DNA similarity. This is also why we do not use techniques such as anchoring, or artificial edges linking the same node in different time frame [14].

In the area of visualization of DNA data Yang et al. [26] study ancestry and ethnic affiliation using DNA genetic markers and make accurate predictions with the help of a Bayesian clustering algorithm. Novembre et al. [23] show that there is a very high correspondence between genetic and geographic distance when they characterized the genetic variation in a sample of 3000 European Individuals.

\section{Dynamic Map Visualization}

Consider the problem of computing a "good" distance measure between a set of known DNA samples, that is based on multiple similarity measures (e.g., NRY and mtDNA), with the goal of creating a "canonical map" of the DNA space spanned by these samples. 
In this map, DNA samples are nodes, two nodes are close to each other if they have a high similarity, and groups of similar nodes are clustered into "countries". Next an unknown DNA sample can be compared to the known ones and then placed on the map, in a way that minimizes its distance to the most similar known samples. In order to do this, we must compute such a "good" distance measure from multiple similarity metrics, for example, by assigning weights to each metric and taking a weighted sum, or some non-linear combination thereof. Once appropriate weights have been assigned we can create the canonical map where we will place unknown DNA samples.

Thus the main problem here is figuring out how to appropriately combine a set of different similarity metrics. Given two different similarity metrics on the same set of DNA samples, a simple way to visualize them is to create two static maps. However, this is not very helpful to the scientists who would like to understand the correspondences and differences between these two metrics, as node positions on the static maps are likely to be unrelated. In addition, color assignment for the countries are random, making it even harder to understand the relationships.

The maps in Figure 1 show the nature of the problem. In Figure 1 (a), 39 subjects are embedded in 2D space based on mtDNA similarity, using multi-dimensional scaling. Figure 1 (b) shows a map obtained from the layout in (a), with subjects clustered using modularity clustering, and the clusters highlighted using the GMap algorithm. Here and later on, we used color palettes from ColorBrewer [5]. In Figure 1 (c), we re-embedded the subjects using a different similarity metric (NRY DNA similarity), independent of Figure 1 (b). Compared with Figure 1 (b), the layout changed significantly. Furthermore, although the same color palette is used, colors are assigned independently, making it even harder to figure out the relationship between Figure 1 (b) and Figure 1 (c). In Figure 1 (d), the embedding of NRY DNA similarity is done to minimize the difference to that of the embedding based on mtDNA similarity measure, making it possible to see that node positions are largely unchanged. However due to the color assignment, it is still difficult to compare with the other map in Figure 1 (b). Finally, in Figure 1 (e), colors are properly matched such that clusters with mostly the same nodes are colored using the same colors. This makes it easy to compare Figures 1 (b) and (e). For example, we can clearly see that two countries in the top left are now merged in a single country.

We seek to create an interactive tool that can smoothly animate from one similarity map to another, by dynamically changing the weight given to each of the two metrics using a slider that implements a linear combination. This approach can be generalized to three metrics with the help of an equilateral triangle in which each interior point corresponds to a convex combination of the three metrics.

To construct such a tool requires us to overcome a number of challenges outlined earlier and illustrated in Figure 1, namely: node stability (node placement should be determined by the underlying similarity metric, while at the same time not differ unnecessarily from one map to the other), cluster stability (nodes from one cluster in one map should be kept in the same cluster in the other map, provided that doing so does not give suboptimal clustering), and color stability (using the same color for countries that share most of their nodes).

Note that the problem of color stability applies equally well to real geographic maps that have been clustered based on two different metrics. For example, Figure 2 shows clusters of countries in Europe, Asia, Africa and Oceania based on two types of international trade metrics. We generate a country graph where each country is a node, and an edge exists between two countries if they trade with each other. Figure 2 (a) is clustered using the total import/export values among countries as edge weights. Figure 2 (b)

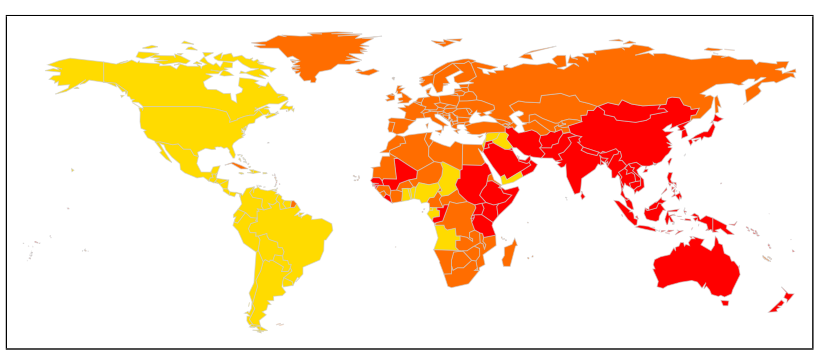

(a)

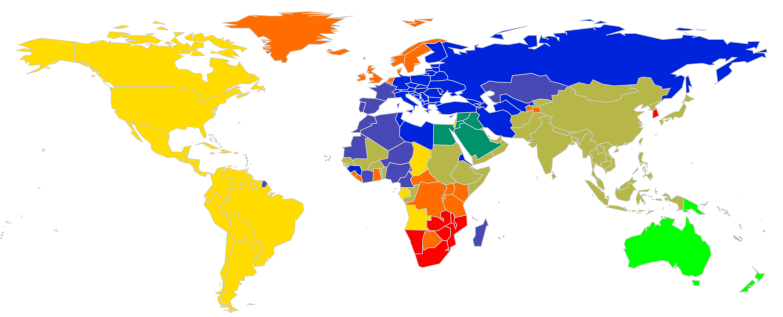

(b)

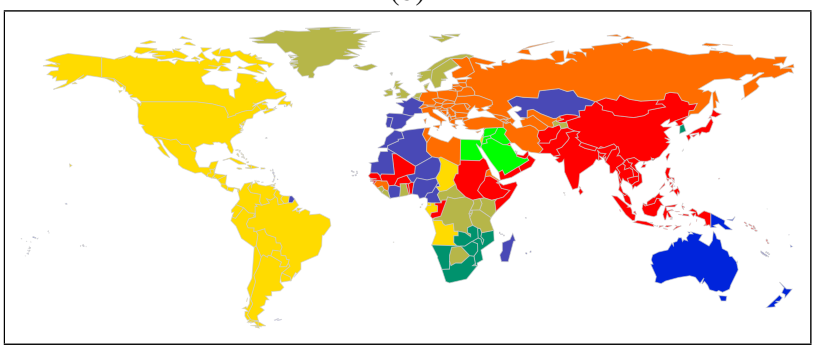

(c)

Figure 2: World trade maps: (a) clustering of countries based on total import/export values between countries; (b) clustering of countries based on total import/export fractions; (c) same clustering as (b), but with color stability.

is clustered using the total import/export fractions ${ }^{1}$ among countries as edge weights. It is difficult to compare the maps in (a-b), because the colors for the clusters are chosen at random. On the other hand, when we apply color-stabilization we obtain the pair of maps (a-c), where clusters in the two maps that share many countries are colored using the same color. Here it is easy to see that the new clusters in Europe match closely with their former colonies in Africa: UK and former colonies in British East Africa such as Kenya, Uganda, Tanzania, as well as France and former colonies Algeria, Morocco, Mauritania. Interestingly, the idea to color a map of Europe and Africa so as to indicate European colonies was used over a hundred years ago in a poster for the German Shipping Company Woermann-Linie; see Fig. 3.

In the following we discuss some of the background algorithms we used as a basic building block for our interactive tools.

\section{Dynamic Map Clustering}

Our dynamic map clustering is based on static graph clustering. With this in mind we briefly review modularity graph clustering for static graphs and then consider the dynamic problem in the context of maps.

\subsection{Modularity clustering}

In graph clustering we look for a partition of the nodes into disjoint subsets so that most of the edges go between nodes within

\footnotetext{
${ }^{1}$ The import/export fraction is a number between 0 to 1 , representing the percentage of import/export from one country to another, as a fraction of its total import/export value.
} 


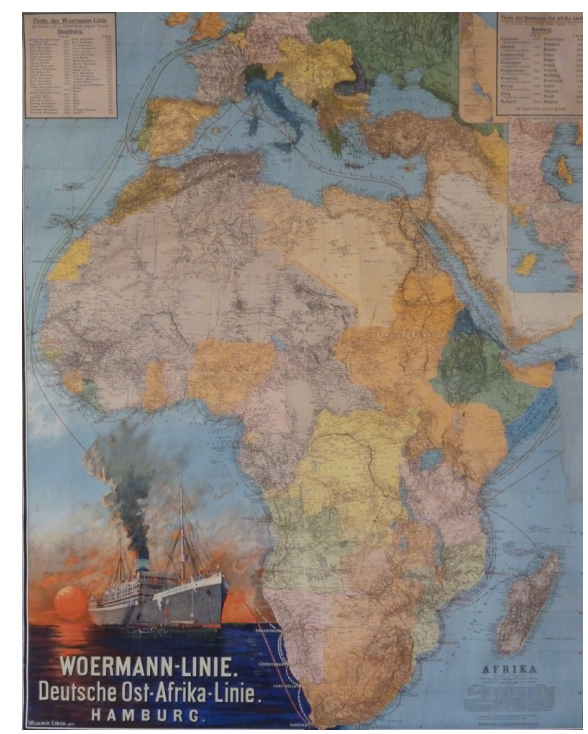

Figure 3: A map of Europe and Africa from a poster for the Woermann-Linie. Note that the colors of countries in Europe match the colors of the colonies in Africa: Mozambique and Portugal, Germany and Namibia, France and Senegal, Belgium and Congo, etc.

a cluster and there are not too many edges between nodes in different clusters. Formally, it is a mapping from the node set to a set of integers $C: V \rightarrow\{1,2, \ldots, k\}$, where $k=|C|$ is the number of clusters. Roughly, clustering algorithms are either divisive or agglomerative. Agglomerative algorithm merge similar nodes or communities recursively, while divisive algorithms detect the intercluster edges and remove them from the network. In both cases, the goal is to optimize some cluster quality measure. One such measure is modularity, defined as a value between -1 and 1 that measures the density of links inside communities compared to links between communities $[27,28]$. For a weighted undirected graph $G=(V, E)$ the modularity is defined as follows:

$$
Q=\frac{1}{2 m} \sum_{i, j \in V}\left[A_{i, j}-\frac{k_{i} k_{j}}{2 m}\right] \delta(C(i), C(j))
$$

Here $A_{i, j}$ is the weight of the edge between $i$ and $j$, where $A_{i, j}$ represents the similarity between $i$ and $j$ (e.g., the number of mutual friends in a social network). The scalar $k_{i}=\sum_{j} A_{i, j}$ is the sum of the weights of the edges attached to node $i$, and $C(i)$ is the cluster node $i$ is assigned to. The $\delta$ - function $\delta(C(i), C(j))$ is 1 if $C(i)=C(j)$ and 0 otherwise, and $m=\frac{1}{2} \sum_{i, j} A_{i, j}$ is the sum of all edge weights.

Finding a clustering which will maximize modularity is known to be a computationally hard problem, as shown by Brandes et al. [3]. We implemented a heuristic algorithm proposed by Blondel et al. [1] (available as the Graphvi z function cluster).

\subsection{Dynamic clustering}

We now consider the clustering problem on dynamic graphs, where the changes are adding/removing nodes, adding/removing edges, and modifications in node weights and edge weights. For the purpose of mental map preservation, we seek to preserve the clustering structure between the iterations as much as possible, provided that doing so does not result in suboptimal clustering.

We propose a simple heuristic to combine the two objectives of modularity and cluster stability for dynamic clustering. This heuristic is a dynamic variation of the agglomerative clustering algorithm of Blondel et al. [1]. Heuristics are a reasonable approach, as the dynamic modularity clustering problem is also NP-Hard [19].

We begin with each node as a singleton. During the first level of clustering, we consider merging only node pairs which belong to the same cluster in the clustering of the previous iteration. When no more node pairs are left for merging, the current clustering is used to construct a "contracted graph" with each cluster as a super node and appropriately adjusted adjacencies and edge weights. We proceed iteratively with the contracted graph as input. The clustering of the previous iteration is explicitly used in the first level and afterwards we apply the algorithm of Blondel et al. [1].

We evaluate the effectiveness of our heuristic with a measure of cluster similarity given by Rand [29]. This measure is based on node-pair clustering as follows. Let $C$ and $C^{\prime}$ denote two clusterings of a graph $G$, and let $S_{11}$ denote the set of pairs that are clustered together in both clusterings, and $S_{00}$ denote the set of pairs that are in different clusters. Then the Rand distance between the two clusterings is given by

$$
\operatorname{rand}\left(C, C^{\prime}\right)=1-\frac{2\left(\left|S_{11}\right|+\left|S_{00}\right|\right)}{n(n-1)}
$$

The value will be 0 if the two clusterings are identical, and 1 if one clustering is a singleton clustering and the other one is that of all nodes in the same cluster.

With the data from Fig. 1, we evaluated the quality between each pair of successive iterations and averaged these values over all successive pairs. Without our heuristic, the average Rand measure was 0.0631 , and with the heuristic, it was 0.0252 . This shows an improvement of a little more than $60 \%$ with the heuristic.

\section{Dynamic Map Coloring}

Color stability, that is, using the same color for countries on the two maps that share most of their nodes, is an essential ingredient in visualizing dynamic maps. In order to maintain color stability, we need to match the best pairs of clusters in different maps.

Given two maps, let $C_{\text {old }}$ and $C_{\text {new }}$ be vectors representing clustering information of these two maps. We have to minimize the number of nodes whose cluster is different in $C_{\text {old }}$ and $C_{\text {new }}$. Let $s\left(C_{\text {old }}, C_{\text {new }}\right)$ be the number of nodes that do not undergo clustering change.

$$
s\left(C_{\text {old }}, C_{\text {new }}\right)=\sum_{u \in V} \delta\left(C_{\text {old }}(u), C_{\text {new }}(u)\right)
$$

$\delta(u, v)=1$ if $u=v$, and 0 otherwise.

The cluster matching problem is to find a permutation $\Pi$ of the clustering $C_{\text {new }}$, such that $\Pi\left(C_{\text {new }}\right)$ maximizes $s\left(C_{\text {old }}, \Pi\left(C_{n e w}\right)\right)$. For example let $C_{\text {old }}=\{1,1,2,2,3\}$ be the clusters assigned to the 5 nodes $v_{1}, v_{2}, v_{3}, v_{4}, v_{5}$; let $C_{\text {new }}=\{2,2,1,3,4\}$ be the new clustering in which $v_{3}$ and $v_{4}$ split into two clusters. Clearly $s\left(C_{\text {old }}, C_{\text {new }}\right)=$ 0 . The optimum matching is the permutation $\Pi:\{1,2,3,4\} \rightarrow$ $\{2,1,4,3\}$. The resulting clustering, $\Pi\left(C_{\text {new }}\right)=\{1,1,2,4,3\}$ gives $s\left(C_{\text {old }}, \Pi\left(C_{\text {new }}\right)\right)=4$

The problem can be modeled with maximum weighted matching (MWM) of a bipartite graph. The corresponding bipartite graph $G_{C}$ has node set $\left\{1,2, \ldots,\left|C_{\text {old }}\right|\right\} \times\left\{1,2, \ldots,\left|C_{\text {new }}\right|\right\}$. The edge weight, $w(i, j)$, corresponds to the number of nodes that are common between cluster $i$ of $C_{\text {old }}$ and cluster $j$ of $C_{\text {new }}$.

$$
w(i, j)=\sum_{u \in V} \phi(i, j, u)
$$

$\phi(i, j, u)=1$ if $C_{\text {old }}(u)=i$ and $C_{\text {new }}(u)=j$.

The maximum weighted bipartite matching of $G_{C}$ gives a matching $\Pi$ between the clusters $C_{\text {old }}$ and $C_{\text {new }}$ that will maximize $s\left(C_{\text {old }}, \Pi\left(C_{\text {new }}\right)\right)$. The MWM for bipartite graphs can be found using the Hungarian algorithm [24]. For bipartite graphs, an efficient implementation of the Hungarian algorithm using Fibonacci heaps [16] runs in $O\left(m n+n^{2} \log n\right)$, where $m$ and $n$ are the number of edges and nodes in $G_{C}$, respectively. If we assume that a cluster 
in the old clustering does not split into more than a constant number of clusters in the new clustering, then $m=O(n)$. This yields a $O\left(n^{2} \log n\right)$ algorithm for MWM. Since $w(i, j)$ are all integers in the range 0 to $|V|$, the algorithm by Gabov and Tarjan algorithm [17] for MWM can be implemented in $O\left(n^{\frac{3}{2}} \log (n|V|)\right)$. In practice, the number of clusters is typically small and our implementation of the Hungarian algorithms is fast enough.

\section{Dynamic Map Layout}

Abstractly, the problem of dynamic maps layout is that of computing node positions, which is related to the well-known readability versus mental map preservation problem for dynamic graph drawing. Traditionally, if given a sequence of graphs, one can compute node positions for the current graph in the sequence by starting with the node positions from the previous graph in the sequence and followed by local node position refinement. One shortcoming of such approaches is that even with the node-position initialization two consecutive graphs in the sequence with very similar topology can have very different drawings, causing node-jumping between frames, and failing to preserve the mental map.

Another dynamic layout approach is to "anchor" some, or all, of the nodes, or to limit node movement by adding artificial edges linking graphs in different time frames [14]. However, such approaches can introduce biases that were not in the data itself, which is undesirable when analyzing highly sensitive real-world data, such as DNA similarity.

We preserve the mental map via a Procrustes transformation of the coordinates of the nodes, after an multidimensional scaling (MDS) layout, initialized with the node positions from the previous iteration. The Procrustes transformation is a well known technique [10], which attempts to find the best alignment of two inputs via rotation, translation, and scaling. Let the layout of the previous frame be $y_{i}, i=1,2, \ldots,|V|$, and the current layout $x_{i}, i=1,2, \ldots,|V|$ (if the node sets in the two layout are different, we take the coordinates for common nodes to derive the transformation). We would like to find a translation vector $b$, scaling value $\rho$ and rotation matrix $T$ such that:

$$
\operatorname{minimize} \sum_{i=1}^{|V|}\left\|y_{i}-\left(\rho T x_{i}+b\right)\right\|^{2} .
$$

The solution to this problem is

$$
T=\left(X Y^{T} Y X^{T}\right)^{1 / 2}\left(Y X^{T}\right)^{-1}, \rho=\frac{\operatorname{tr}\left(\left(X Y^{T} Y X^{T}\right)^{1 / 2}\right)}{\operatorname{tr}\left(X X^{T}\right)},
$$

where $X$ is the $2 \times|V|$ matrix of $x_{i}$ 's. The translation vector is $b=$ $\frac{1}{|V|}\left(\sum_{i=1}^{|V|} y_{i}-\rho T\left(\sum_{i=1}^{|V|} x_{i}\right)\right)$. The minimal value of (5),

$$
1-\frac{\operatorname{tr}\left(\left(X^{T} Y Y^{T} X\right)^{1 / 2}\right)}{\operatorname{tr}\left(X^{T} X\right) \operatorname{tr}\left(Y^{T} Y\right)}
$$

is known as the Procrustes Statistic.

Note that if we do not restrict $T$ to be an orthogonal rotation matrix, then $\rho$ is subsumed by $T$ and we have a general affine transformation, with $T=Y X^{T}\left(X X^{T}\right)^{-1}$.

The difference between the Procrustes transformation, and a general affine transformation, is that the Procrustes transformation preserves the relative distance between nodes. Figure 4 shows the pipeline of how the transformation is applied to a sequence of graphs.

We experimented with both the Procrustes and affine transformations, and found that although affine transformation are better at minimizing the differences between two frames, the Procrustes

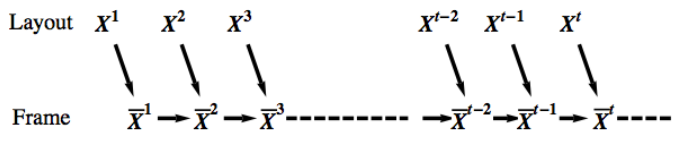

Figure 4: The affine transformation pipeline. $X^{t}$ denotes the layout at time $t$. This layout is transformed to fit the previous frame $\bar{X}^{t-1}$ as close as possible to give the current frame $\bar{X}^{t}$, and the frames are what the user sees.

transformation is good enough, but with the added benefit of preserving relative distances. However, when using either in dynamic map visualization involving many frames, we found an unexpected problem: On inputs where the first frame is tall and thin, and the second frame is a square with the same height as the first, the image size after transformation shrinks as we progress through the time steps, even beyond the second frame. Further analysis confirms that although each transformation is strictly derived from the current layout, and the previous frame, the Procrustes and affine transformations have a memory of layouts that goes all the way to the first layout. This can be proven as the following theorem, which relates the layout at time $t, X^{t}$, and the frame at time $t, \bar{X}^{t}$, obtained by the attempt to "fit" $X^{t}$ with the previous frame $\bar{X}^{t-1}$ :

Theorem 1. Let $\rho(Y, X)$ be the scaling factor for the Procrustes transformation of points $\left\{x_{1}, \ldots, x_{|V|}\right\}$ to points $\left\{y_{1}, \ldots, y_{|V|}\right\}$. Then

$$
\rho\left(\bar{X}^{t-1}, X^{t}\right)=\rho\left(X^{t-1}, X^{t}\right) \rho\left(\bar{X}^{t-2}, X^{t-1}\right) .
$$

Proof. Denote the translation vector and transformation matrix for the Procrustes transformation of points $\left\{x_{1}, \ldots, x_{|V|}\right\}$ to fit points $\left\{y_{1}, \ldots, y_{|V|}\right\}$ as $b(Y, X)$ and $T(Y, X)$. Then by definition, $\rho\left(\bar{X}^{t-1}, X^{t}\right)$ is the solution of

$$
\operatorname{minimize}_{\rho, T, b} \sum_{i=1}^{|V|}\left\|\bar{x}_{i}^{t-1}-\left(\rho T x_{i}^{t}+b\right)\right\|^{2} .
$$

As $\bar{X}^{t-1}$ is itself a Procrustes transformation of $X^{t-1}$, it must be of the form

$$
\bar{x}_{i}^{t-1}=\rho\left(\bar{X}^{t-2}, X^{t-1}\right) T_{2} x_{i}^{t-1}+b_{2}
$$

with $T_{2}$ an $2 \times 2$ orthogonal matrix and $b_{2} \in R^{2}$. Substituting in the previous equation shows that $\rho\left(\bar{X}^{t-1}, X^{t}\right)$ is the solution of

$$
\operatorname{minimize}_{\rho, T, b} \sum_{i=1}^{|V|}\left\|\rho\left(\bar{X}^{t-2}, X^{t-1}\right) T_{2} x_{i}^{t-1}+b_{2}-\left(\rho T x_{i}^{t}+b\right)\right\|^{2} .
$$

Since the 2-norm is invariant under orthogonal transformation, the above is the same as

$$
\begin{gathered}
\operatorname{minimize}_{\rho, T, b} \sum_{i=1}^{|V|} \| x_{i}^{t-1}-\left(\frac{\rho}{\rho\left(\bar{X}^{t-2}, X^{t-1}\right)} T_{2}^{T} T x_{i}^{t}\right. \\
\left.+T_{2}^{T} \frac{b-b_{2}}{\rho\left(\bar{X}^{t-2}, X^{t-1}\right)}\right) \|^{2} .
\end{gathered}
$$

Because here $T_{2}^{T} T$ represents any $2 \times 2$ orthogonal matrix for a suitably chosen $T$, this means that $\frac{\rho\left(\bar{X}^{t-1}, X^{t}\right)}{\rho\left(\bar{X}^{t-2}, X^{t-1}\right)}$ must be a solution of

$$
\operatorname{minimize}_{\rho, T, b} \sum_{i=1}^{|V|}\left\|x_{i}^{t-1}-\left(T x_{i}^{t}+b\right)\right\|^{2}
$$

in other words,

$$
\frac{\rho\left(\bar{X}^{t-1}, X^{t}\right)}{\rho\left(\bar{X}^{t-2}, X^{t-1}\right)}=\rho\left(X^{t-1}, X^{t}\right)
$$

or

$$
\rho\left(\bar{X}^{t-1}, X^{t}\right)=\rho\left(X^{t-1}, X^{t}\right) \rho\left(\bar{X}^{t-2}, X^{t-1}\right) .
$$


The same theorem applies to affine scaling. Applying this theorem recursively gives

$$
\rho\left(\bar{X}^{t-1}, X^{t}\right)=\Pi_{i=2}^{t} \rho\left(X^{i-1}, X^{i}\right) .
$$

Hence any scaling difference between two previous layouts will have a multiplicative effect on the current layout. For example, if the first layout $X^{1}$ is $20 \%$ smaller than $X^{2}$, then a Procrustes transformation to any subsequent layout will cause a down-scaling by a multiple of 0.8 . Over time, for some cases the scaling can drift far from 1, causing problems such as severe label overlap. Since for a smooth visualization, it is necessary to preserve the mental map only among a few nearby frames (rather than over the entire sequence of frames), we took the approach of combining the transformed coordinates and the original coordinates with a parameter $0<s<1$, where $s=1$ yields the transformed coordinates and $s=0$ the original coordinates. In our implementation we use $s=0.5$.

\subsection{Evaluating layout stability}

To evaluate the different layout stability approaches, we compare the trajectories of a set of randomly selected nodes from the dataset in Fig. 1. Fig. 5 (top) shows such node trajectories, where the position of a node in the new graph is obtained by an independent MDS computation of the two layouts. Fig. 5 (middle) shows the node trajectories, when using an MDS layout of the current frame, where the position of each node is initialized with the position obtained from the previous frame. Finally, Fig. 5 (bottom) shows node trajectories, where the position of each node is initialized with the position obtained from the previous iteration and combined with a Procrustes transformation to fit the previous frame.

Fig. 6 compares two sets of maps: one drawn without affine transformation (left) and one drawn with affine transformation. The maps were computed by interpolating between the mtDNA and NRY DNA maps from Fig. 1 with weights 30, 50, 70 and 90 for $w$ as explained in Section 7. Note that the differences between the left and right maps in Fig. 6 grows with each iteration. That is, each successive pair of frames shows a greater difference than its predecessor. This is because the changes due to the affine transformation are cumulative since it is applied at every stage. Consequently, the final results in the sequences are not affine transformations of each other.

In all cases we experimented with, the last strategy was the best one, its trajectories the least jittery. We quantify these strategies by computing the average node-travel distance per frame (over all nodes in the graph, not just the random sample shown in the Figure), and in this example the distances traveled are 21.41, 13.19 and 8.43 pixels, respectively. This confirms that there are non-trivial improvements when we use the initial nodes position together with a Procrustes transformation.

\section{The Dynamic Maps System}

We have implemented an interactive system which allows for the exploration and visualization of the effect of linear interpolation between two similarity metrics (represented by a pair of adjacency matrices). We obtain smooth transitions from one matrix to another, and which the viewer can manipulate or just watch. Let $w$ be a weight in the range from 0 to 100 and let $M_{1}$ and $M_{2}$ be the two input matrices. We compute the weighted matrix $M_{w}=$ $\frac{w \times M_{1}+(100-w) \times M_{2}}{100}$. The weighted matrix is then passed on to the module that computes the dynamic map clustering, then to the module that computes the dynamic map coloring, and finally to the module that computes the dynamic map layout.

The embedding of the graph was done using Graphviz [13]. In particular, we used the neato layout engine of graphviz. In

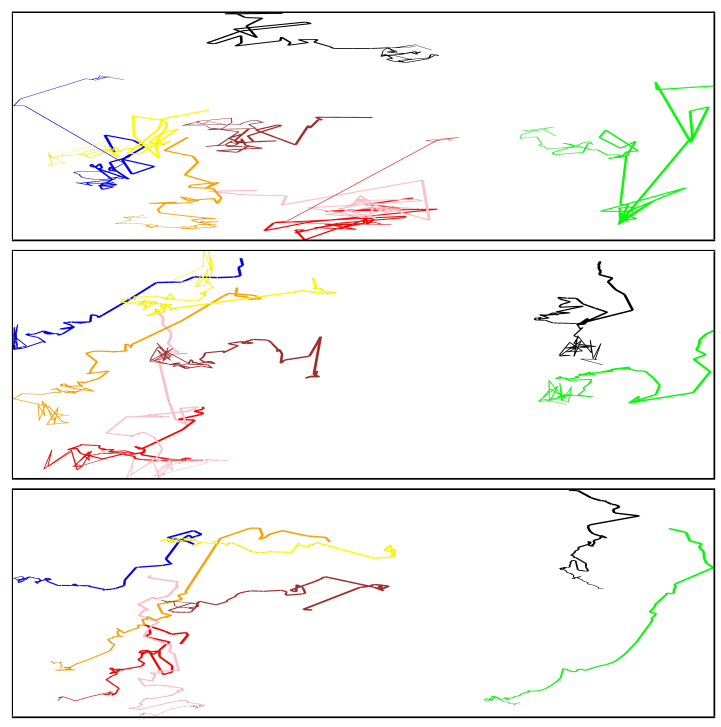

Figure 5: Trajectories of randomly selected nodes with three different layout stability methods. (Top) independent layout with average distance traveled 21.41; (Middle) layout initialized with positions from the previous frame with average distance traveled 13.19; (Bottom) initialized positions and Procrustes transformation, with average distance traveled 8.43 .

addition to neato, the gvpr scripting language is used for manipulating graphs. Finally, GMap [22], available as part of Graphviz provides most of the geographic map functionality.

We briefly consider another example with a different type of underlying data, which shows that our system is flexible and can be applied to examples other than the motivating DNA similarity analysis. Figure 7 shows a bit of the evolution of the collaboration graph of authors who published research papers in the Symposium on Graph Drawing in 2000, 2001 and 2002. Nodes are authors and two authors are connected by an edge if they co-authored a paper. The edge weight is the number of co-authored papers. We used the system described in the paper directly, applying the layout, cluster and color stability strategies proposed in this paper, we can obtain maps that allow us to easily compare author clusters evolution over time. Movies of the system in action and a collection of images can be found at http://cs.arizona. edu/ sankar/dynamap.

\section{Conclusion and Future Work}

In this paper we presented a practical approach for visualizing dynamic relational data and multiple relationships on the same data, using a geographic map metaphor. We suggested a heuristic to address the challenge of dynamic cluster stability, proposed a strategy to preserve color stability, and analyzed the use of affine transformations to improve layout stability. We applied these results in an interactive tool for DNA visualization.

We modeled the color stability as a maximum weighted matching of a bipartite graph. There are alternative ways to model the cluster stability problem that deserve further investigation. The stable marriage problem could be used to match clusters in $C_{\text {old }}$ and $C_{n e w}$. Each cluster $i$ in $C_{\text {old }}$ has a preference list $\left\{j_{1}, j_{2}, j_{3} \cdots j_{n}\right\}$, where $j_{k}$ 's are the clusters in $C_{n e w}$. The cluster $j_{k}$ is preferred over $j_{l}$ in the list of $i$ if and only if $w\left(i, j_{k}\right) \geq w\left(i, j_{l}\right)$. The Gale-Shapley algorithm for stable marriage runs in $O(m)$ [18], however, the algorithm is not symmetric. That is, the solution obtained with respect to $C_{\text {old }}$ is not necessarily the same as that obtained with respect to $C_{n e w}$. If the two solutions are indeed different, we could compute both and take the better of the two (in terms of numbers of matches).

It is also desirable for the majority of the elements in each cluster to remain in the same cluster. As this property cannot be guaranteed for all clusters, we can instead maximize the number of clusters 

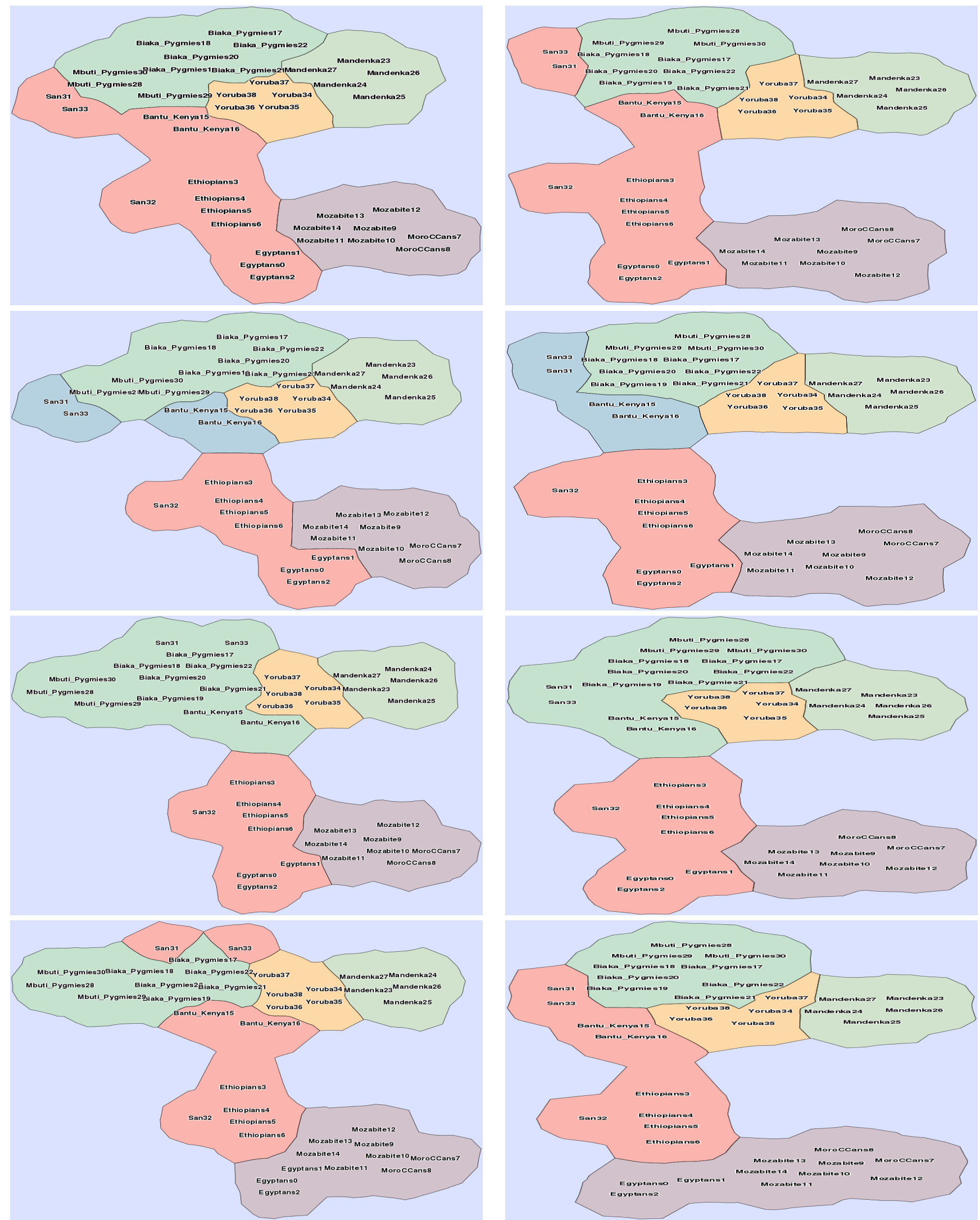

Figure 6: A sequence of maps obtained by combining mtDNA and NRY DNA similarity metrics. Note that in both sequences the clusters match well and the colors of the clusters also match well. The sequence in the left and right employ different node stabilization: (Left) sequence obtained without affine transformation; (Right) a sequence obtained with affine transformation. Note that although both this Figure and Figure 1 are part of the same animation, this Figure is the result of quite different weight values from that of Figure 1, thus the figures do not look similar. 


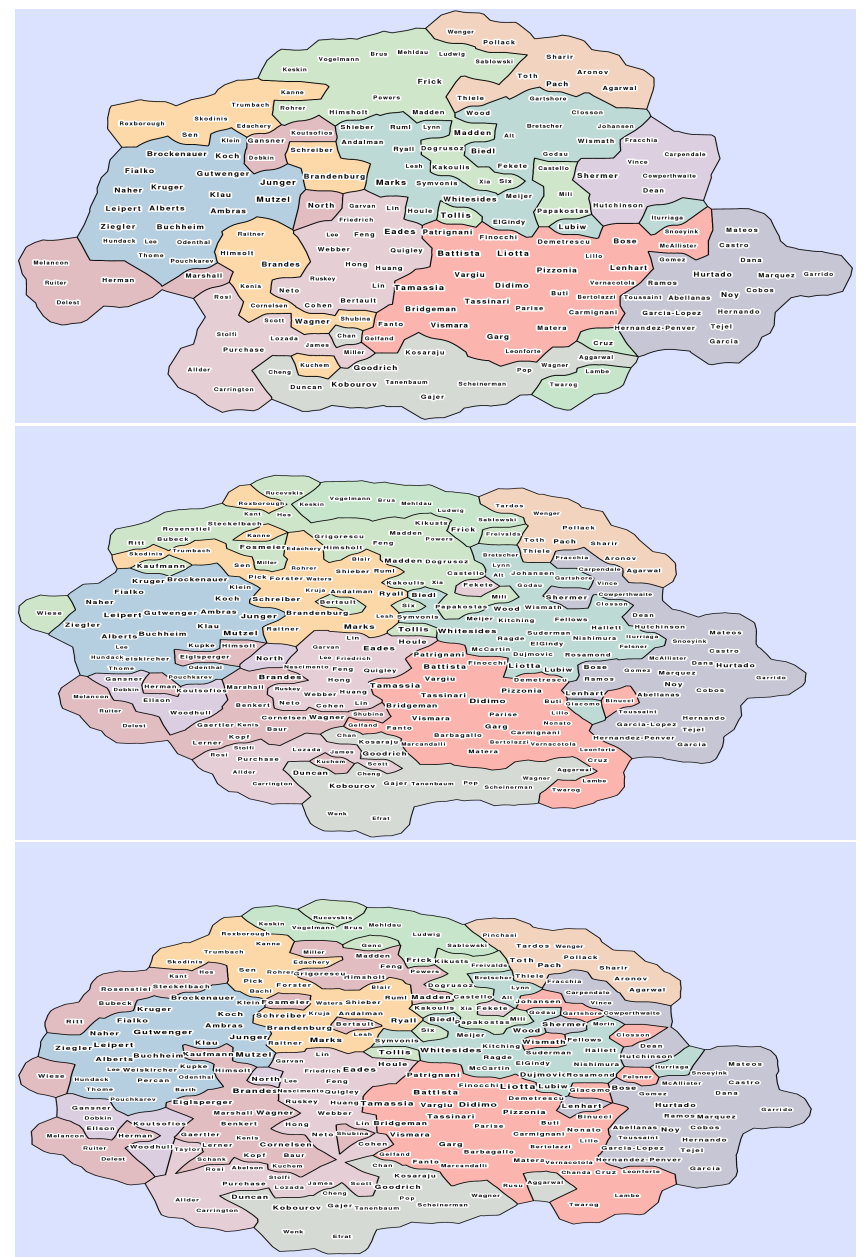

Figure 7: A sequence of GD collaboration graphs: 2000, 2001 and 2002.

for which this property holds. Let $k$ be the number of clusters in $C_{\text {old }}$. The objective function to optimize is $Z=\sum_{0 \leq i \leq k} z_{i}$, where $z_{i}$ $=1$ if the majority of the elements in cluster $i$ in the old clustering remains in cluster $i$ in the new clustering, and 0 otherwise. $Z$ may be optimized using a simple greedy algorithm.

We are currently testing the system with the help of forbearing biologists who are using multiple DNA-similarity metrics. In addition to a formal evaluation of the effectiveness of our method, we would also like to be able to combine more than 2 similarity metrics, to allow for more general ways to combine multiple similarity metrics, and to deploy the system in a generic dynamic data setting.

\section{Acknowledgements}

We thank Michael Hammer and Nirav Merchant from the Bio5 Institute for introducing us to the DNA-similarity problem and for their patience in explaining the various similarity metrics and the underlying biology.

\section{References}

[1] V. Blondel, J. Guillaume, R. Lambiotte, and E. Lefebvre. Fast unfolding of communities in large networks. Journal of Stat. Mechanics: Theory and Experiment, 2008:P10008, 2008.

[2] U. Brandes and S. R. Corman. Visual unrolling of network evolution and the analysis of dynamic discourse. In IEEE INFOVIS' 02 , pages 145-151, 2002.

[3] U. Brandes, D. Delling, M. Gaertler, R. Görke, M. Hoefer, Z. Nikoloski, and D. Wagner. On finding graph clusterings with maximum modularity. In $W^{\prime} 07$, pages 121-132, 2007.
[4] U. Brandes and D. Wagner. A Bayesian paradigm for dynamic graph layout. In 5th Symp. on Graph Drawing (GD), pages 236-247, 1998.

[5] C. Brewer. ColorBrewer - Color Advice for Maps. www . colorbrewer2. org.

[6] M. Burch and S. Diehl. Timeradartrees: Visualizing dynamic compound digraphs. Computer Graphics Forum, 27(3):823-830, 2008.

[7] H. Byelas and A. Telea. Visualization of areas of interest in software architecture diagrams. In ACM Symp. on Software Visualization (SoftVis'06), pages 105-114, 2006.

[8] C. Collins, G. Penn, and S. Carpendale. Bubble sets: Revealing set relations with isocontours over existing visualizations. IEEE TVCG, 15(6): 1009-1016, 2009.

[9] P. F. Cortese, G. D. Battista, A. Moneta, M. Patrignani, and M. Pizzonia. Topographic visualization of prefix propagation in the internet. IEEE TVCG, 12:725-732, 2006.

[10] T. F. Cox and M. A. A. Cox. Multidimensional Scaling. Chapman and Hall/CRC, 2000.

[11] W. Cui, Y. Wu, S. Liu, F. Wei, M. X. Zhou, and H. Qu. Contextpreserving, dynamic word cloud visualization. Computer Graphics and Applications, 30:42-53, 2010.

[12] S. Diehl and C. Görg. Graphs, they are changing. In 10th Symp. on Graph Drawing (GD), pages 23-30, 2002.

[13] J. Ellson, E. Gansner, E. Koutsofios, S. North, and G. Woodhull Graphviz and dynagraph-static and dynamic graph drawing tools. Graph Drawing Software, pages 127-148, 2003.

[14] C. Erten, P. J. Harding, S. G. Kobourov, K. Wampler, and G. V. Yee. GraphAEL: Graph Animations with Evolving Layouts. In 11th Symp. on Graph Drawing, pages 98-110, 2003.

[15] S. I. Fabrikant, D. R. Montello, and D. M. Mark. The distancesimilarity metaphor in region-display spatializations. IEEE Computer Graphics \& Application, 26:34-44, 2006.

[16] M. L. Fredman and R. E. Tarjan. Fibonacci heaps and their uses in improved network optimization algorithms. J. ACM, 34:596-615, July 1987.

[17] H. N. Gabow and R. E. Tarjan. Faster scaling algorithms for network problems. SIAM J. Comput., 18:1013-1036, October 1989.

[18] D. Gale and L. S. Shapley. College admissions and the stability of marriage. The American Mathematical Monthly, 69(1):9-15, 1962.

[19] R. Görke, P. Maillard, C. Staudt, and D. Wagner. Modularity-driven clustering of dynamic graphs. In 9th Symp. on Experimental Algorithms, pages 436-448, 2010.

[20] Herman, G. Melançon, and M. S. Marshall. Graph visualization and navigation in information visualization: A survey. IEEE Transactions on Visualization and Computer Graphics, 6(1):24-43, 2000.

[21] D. Holten and J. J. van Wijk. Visual comparison of hierarchically organized data. Computer Graphics Forum, 27(3):759-766, 2008.

[22] Y. Hu, E. Gansner, and S. Kobourov. Visualizing Graphs and Clusters as Maps. IEEE Computer Graphics and Applications, 99(1):54-66, 2010.

[23] J. Novembre et al. Genes mirror geography within Europe. Nature, 456(7218):98-101, 2008.

[24] H. W. Kuhn. The hungarian method for the assignment problem. Naval Research Logistics Quarterly, 2(1-2):83-97, 1955.

[25] D. Mashima, S. G. Kobourov, and Y. F. Hu. Visualizing dynamic data with maps. In IEEE Pacific Visualization Symposium, pages 155-162, 2011.

[26] N. Yang et al. Examination of ancestry and ethnic affiliation using highly informative diallelic DNA markers: application to diverse and admixed populations and implications for clinical epidemiology and forensic medicine. Human genetics, 118(3):382-392, 2005.

[27] M. E. J. Newman. Analysis of weighted networks. Phys. Rev. E, 70(5):056131, Nov 2004.

[28] M. E. J. Newman and M. Girvan. Finding and evaluating community structure in networks. Phys. Rev. E, 69(2):026113, Feb 2004.

[29] W. M. Rand. Objective criteria for the evaluation of clustering methods. J. of the American Statistical Association, pages 846-850, 1971.

[30] P. Simonetto, D. Auber, and D. Archambault. Fully automatic visualisation of overlapping sets. Computer Graphics Forum, 28:967-974, 2009. 